\title{
Crazy Like a Fox? Are Leaders with Reputations for Madness More Successful at International Coercion? - CORRIGENDUM
}

Roseanne W. McManus

https://doi.org/10.1017/S0007123419000401, Published by Cambridge University Press, 9 December 2019.

Keywords: conflict bargaining; resolve; credibility; reputation; madman theory; leaders in international conflict; corrigendum

The author apologizes for an error in the coding of the variable "Percentage of Capabilities Held by State A." This variable appears in all of the main regressions and, therefore, correcting it changes all of these regression results. Most of these changes do not affect the substantive conclusions, but this corrigendum presents the correct results for all tables and graphs in the order of the paper and appendix. 
Table 2 should be replaced with the following:

Table 2. Main results

\begin{tabular}{|c|c|c|c|c|}
\hline & $\begin{array}{c}1 \\
\text { Initiation }\end{array}$ & $\begin{array}{c}2 \\
\text { Initiation }\end{array}$ & $\begin{array}{c}3 \\
\text { Reciprocation }\end{array}$ & $\begin{array}{c}4 \\
\text { Reciprocation }\end{array}$ \\
\hline Continuous madness rep., A & $\begin{array}{l}0.213^{\star \star} \\
(0.083)\end{array}$ & & $\begin{array}{l}0.264^{\star \star \star} \\
(0.065)\end{array}$ & \\
\hline Continuous madness rep., B & $\begin{array}{l}0.379^{\star \star \star \star} \\
(0.061)\end{array}$ & & $\begin{array}{c}0.032 \\
(0.123)\end{array}$ & \\
\hline Strong madness reputation, $A$ & & $\begin{array}{c}0.177 \\
(0.208)\end{array}$ & & $\begin{array}{l}0.436^{\star \star \star} \\
(0.156)\end{array}$ \\
\hline Slight madness reputation, $A$ & & $\begin{array}{c}0.100 \\
(0.069)\end{array}$ & & $\begin{array}{c}-0.178 \\
(0.222)\end{array}$ \\
\hline Strong madness reputation, B & & $\begin{array}{l}0.902^{\star \star \star} \\
(0.140)\end{array}$ & & $\begin{array}{l}-0.563^{\star \star \star} \\
(0.201)\end{array}$ \\
\hline Slight madness reputation, B & & $\begin{array}{l}0.170^{\star *} \\
(0.075)\end{array}$ & & $\begin{array}{c}-0.210 \\
(0.183)\end{array}$ \\
\hline Recent MID initiations, $A$ & $\begin{array}{l}0.206^{\star \star \star} \\
(0.031)\end{array}$ & $\begin{array}{l}0.201^{\star \star \star} \\
(0.031)\end{array}$ & $\begin{array}{r}-0.032 \\
(0.099)\end{array}$ & $\begin{array}{r}-0.026 \\
(0.103)\end{array}$ \\
\hline Recent MID initiations, $B$ & $\begin{array}{l}0.094^{\star \star \star} \\
(0.034)\end{array}$ & $\begin{array}{l}0.080^{\star *} \\
(0.035)\end{array}$ & $\begin{array}{c}0.058 \\
(0.089)\end{array}$ & $\begin{array}{c}0.091 \\
(0.095)\end{array}$ \\
\hline Military capabilities, A & $\begin{array}{c}1.005 \\
(0.790)\end{array}$ & $\begin{array}{c}0.893 \\
(0.805)\end{array}$ & $\begin{array}{c}-0.297 \\
(2.038)\end{array}$ & $\begin{array}{c}-0.114 \\
(2.055)\end{array}$ \\
\hline Military capabilities, B & $\begin{array}{l}2.479^{\star \star \star \star} \\
(0.680)\end{array}$ & $\begin{array}{l}2.521^{\star \star \star} \\
(0.682)\end{array}$ & $\begin{array}{c}-2.209 \\
(1.566)\end{array}$ & $\begin{array}{r}-2.903^{\star} \\
(1.584)\end{array}$ \\
\hline$\%$ Military cap. held by $\mathrm{A}$ & $\begin{array}{l}0.352^{\star \star \star} \\
(0.099)\end{array}$ & $\begin{array}{l}0.358^{\star \star \star} \\
(0.099)\end{array}$ & $\begin{array}{c}-0.115 \\
(0.247)\end{array}$ & $\begin{array}{c}-0.170 \\
(0.242)\end{array}$ \\
\hline Democracy, A & $\begin{array}{c}0.092 \\
(0.058)\end{array}$ & $\begin{array}{c}0.069 \\
(0.057)\end{array}$ & $\begin{array}{c}-0.271 \\
(0.204)\end{array}$ & $\begin{array}{c}-0.203 \\
(0.203)\end{array}$ \\
\hline Democracy, B & $\begin{array}{l}0.136^{\star \star} \\
(0.060)\end{array}$ & $\begin{array}{l}0.134^{\star *} \\
(0.061)\end{array}$ & $\begin{array}{c}0.023 \\
(0.166)\end{array}$ & $\begin{array}{c}-0.002 \\
(0.158)\end{array}$ \\
\hline Joint democracy & $\begin{array}{l}-0.501^{\star \star \star} \\
(0.107)\end{array}$ & $\begin{array}{l}-0.487^{\star \star \star} \\
(0.107)\end{array}$ & $\begin{array}{c}-0.115 \\
(0.310)\end{array}$ & $\begin{array}{c}-0.167 \\
(0.306)\end{array}$ \\
\hline Contiguity & $\begin{array}{l}0.537^{\star \star \star} \\
(0.072)\end{array}$ & $\begin{array}{l}0.545^{\star \star \star} \\
(0.073)\end{array}$ & $\begin{array}{l}0.386^{\star \star \star} \\
(0.134)\end{array}$ & $\begin{array}{l}0.311^{\star \star} \\
(0.137)\end{array}$ \\
\hline Distance & $\begin{array}{l}-0.118^{\star \star \star} \\
(0.025)\end{array}$ & $\begin{array}{l}-0.119^{\star \star \star} \\
(0.024)\end{array}$ & $\begin{array}{c}0.033 \\
(0.038)\end{array}$ & $\begin{array}{c}0.044 \\
(0.041)\end{array}$ \\
\hline Dyad length & $\begin{array}{l}0.670^{\star \star \star} \\
(0.082)\end{array}$ & $\begin{array}{l}0.631^{\star \star \star} \\
(0.078)\end{array}$ & & \\
\hline Peace years & $\begin{array}{l}-0.042^{\star \star \star} \\
(0.004)\end{array}$ & $\begin{array}{l}-0.043^{\star * *} \\
(0.004)\end{array}$ & & \\
\hline Peace years squared & $\begin{array}{l}0.001^{\star \star \star} \\
(0.000)\end{array}$ & $\begin{array}{l}0.001^{\star \star \star} \\
(0.000)\end{array}$ & & \\
\hline Peace years cubed & $\begin{array}{c}-0.000^{\star \star \star} \\
(0.000)\end{array}$ & $\begin{array}{c}-0.000^{\star \star \star} \\
(0.000)\end{array}$ & & \\
\hline First act hostility & & & $\begin{array}{c}-0.044 \\
(0.153)\end{array}$ & $\begin{array}{c}-0.065 \\
(0.149)\end{array}$ \\
\hline Constant & $\begin{array}{l}-2.876^{\star \star \star} \\
(0.135)\end{array}$ & $\begin{array}{l}-2.841^{\star \star \star} \\
(0.131)\end{array}$ & $\begin{array}{c}-0.124 \\
(0.602)\end{array}$ & $\begin{array}{c}0.061 \\
(0.596)\end{array}$ \\
\hline Observations & 62,384 & 62,384 & 759 & 759 \\
\hline
\end{tabular}

Note: Models 1-2 are probits predicting MID Initiation, with standard errors clustered by dyad. Models 3-4 are probits predicting Reciprocation, with standard errors clustered by State A. The madness reputation variables are lagged by one year. ${ }^{*} p<0.10,{ }^{* *} p<0.05,{ }^{* * *} p$ $<0.01$.

One change to the text is necessary due to the changes to the models in Table 2. In the discussion of the results, the second part of the sentence, "In contrast, leaders with slight madness reputations are only 1.4 times more likely to be targeted than leaders with no madness reputation, and this difference is only significant at the 94 per cent confidence level," should be removed because this difference now surpasses the 95 per cent confidence threshold. 
The predicted probabilities in Figure 1 also change, although the changes are difficult to discern with the human eye. The corrected Figure 1 appears below:
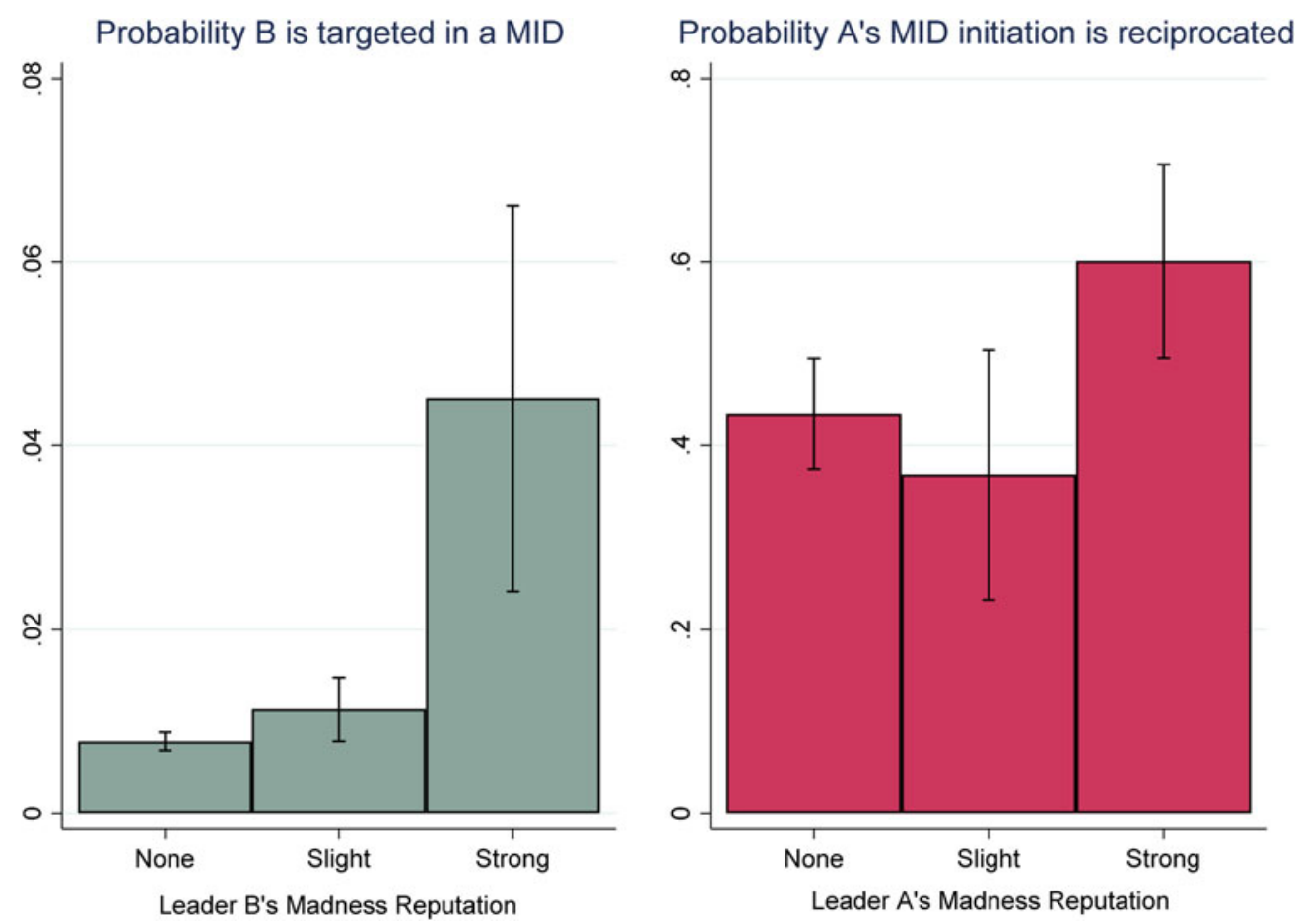

Figure 1. Predicted probabilities from Models 2 and 4

Note: the figure shows average predicted probabilities, produced by calculating the predicted probability for every observation and averaging. The lines represent 95 per cent confidence bounds. 
The changes to Figure 2 are much more substantive. Figure 2 should be replaced with the following:
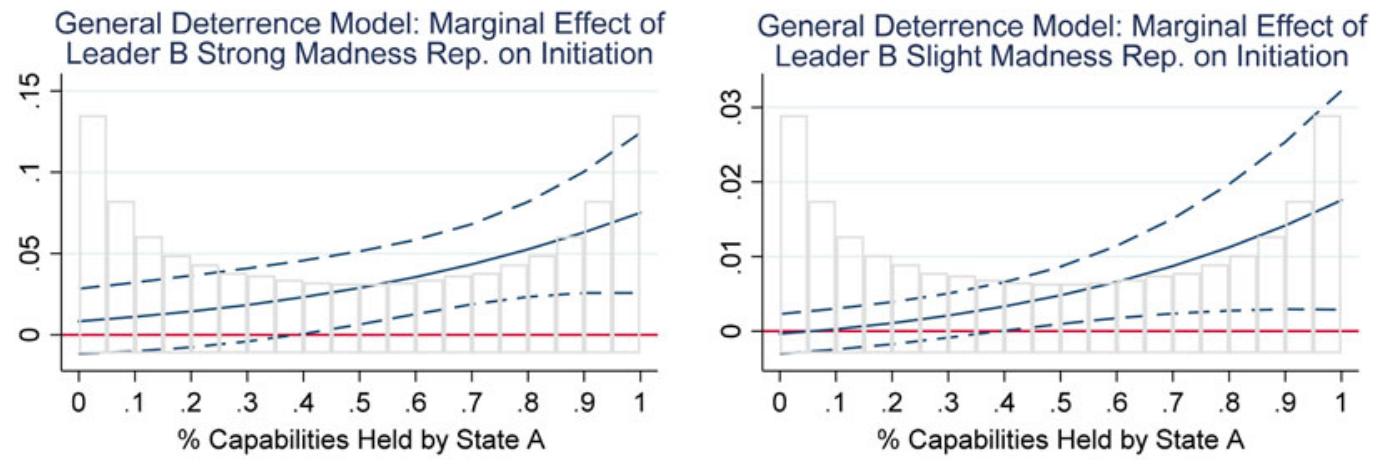

Crisis Bargaining Model: Marginal Effect of Leader A Strong Madness Rep. on Reciprocation

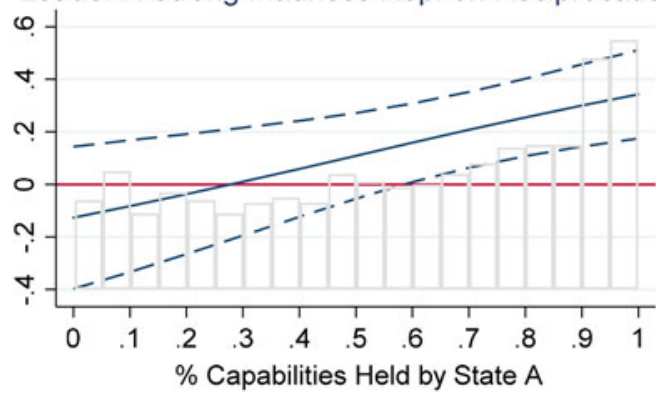

Crisis Bargaining Model: Marginal Effect of Leader A Slight Madness Rep. on Reciprocation

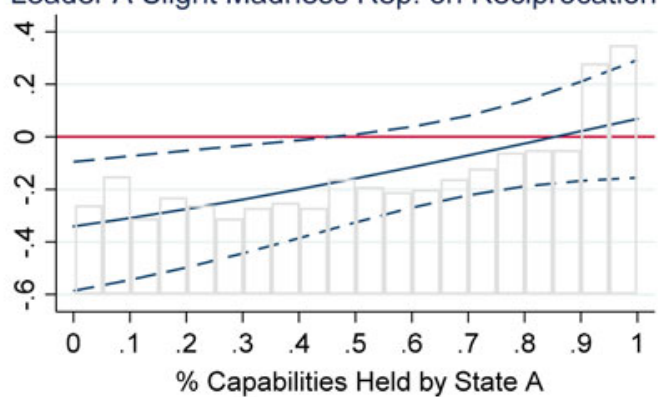

Figure 2. Marginal effects from interactions with relative capabilities

Note: these are average marginal effects. The dotted lines represent 95 per cent confidence bounds. The histograms in the background show the distribution of relative military capabilities.

As a result of this correction, the text in the "Military capabilities interaction" section should be replaced with the following:

"Hypothesis 4 can be tested by interacting the madness reputation indicators with relative military capabilities - specifically, with the percentage of military capabilities in the dyad held by State A. Figure 2 shows marginal effects plots from the interactions. First, analyzing the deterrence regression plots in the top row of Figure 2, the marginal effects of both Strong Madness Reputation and Slight Madness Reputation on the probability of deterrence failure increase as Leader B's relative power weakens (i.e., the percentage of capabilities held by State A increases). This does not support Hypothesis 4.

Turning to the bottom row of Figure 2, we analyze the crisis bargaining regression. The marginal effects from this regression provide support for Hypothesis 4. As State A's military capabilities increase relative to State B, Strong Madness Reputation has an increasingly large detrimental effect on the probability of reciprocation, while the effect of Slight Madness Reputation changes from helpful to insignificant. Therefore, the support for Hypothesis 4 is mixed, which might reflect a greater commitment problem within dyads that are already experiencing conflict.

Figure 2 also shows that perceived madness can actually be beneficial, as the bottom right graph shows that Slight Madness Reputation has a negative and significant marginal effect on 
the probability of MID reciprocation when State A holds less than about 40 percent of capabilities in the dyad."

Due to the changes to Figure 2, some changes to the text in the introduction and conclusion are also necessary. In the introduction, the text beginning with, "I find that perceived madness is clearly harmful to general deterrence," should be amended as follows (with changes and additions in bold):

"I find that perceived madness is clearly harmful to general deterrence and typically has a harmful or insignificant effect in crisis bargaining. However, it may be helpful in crisis bargaining under certain conditions, particularly when the reputation for madness is slight and is coupled with relatively weak military power. My analysis suggests that the harmful effect of perceived madness, at least in the context of crisis bargaining, results from a commitment problem."

The first two paragraphs of the conclusion should be amended as follows (with changes and additions in bold):

"Overall, my findings suggest little support for the Madman Theory. For general deterrence, the effect of perceived madness is purely harmful. In crisis bargaining, the effect of a strong madness reputation seems to be generally harmful or at least unhelpful, but it does appear that a slight madness reputation can be beneficial when a leader's country does not have too much relative military power. In sum, therefore, the effect of perceived madness is more often harmful than helpful. The main apparent beneficiaries of a madness reputation are militarily weak leaders who are perceived as only slightly mad - not necessarily those we would be most likely to think of as 'madmen'.

Why does a reputation for madness often undermine coercive success? My findings suggest that the inability of perceived madmen to make credible commitments to peace may be key. I find that greater relative military power, which increases the commitment problem, causes the impact of a madness reputation to become more detrimental in crisis bargaining (although not for general deterrence). This suggests that when a reputation for madness prevents a leader from credibly committing not to attack in the future, adversaries are more likely to resist the leader firmly. Thus, my findings are in line with research that emphasizes mistrust and the commitment problem as causes of war."

Finally, most of the tables in the appendix have changed slightly, although none of these changes affect the conclusions drawn in the text of the article. The corrected tables appear below in order: 
Table A2: Summary Statistics from General Deterrence (MID Initiation) Dataset

\begin{tabular}{|c|c|c|c|c|c|}
\hline Variable & Mean & SD & Median & Min & Max \\
\hline Initiation & 0.007 & 0.083 & 0 & 0 & 1 \\
\hline Continuous Madness Rep., A & 0.023 & 0.134 & 0 & 0 & 6.461 \\
\hline Continuous Madness Rep., B & 0.023 & 0.134 & 0 & 0 & 6.461 \\
\hline Strong Madness Reputation, Leader A & 0.005 & 0.067 & 0 & 0 & 1 \\
\hline Slight Madness Reputation, Leader A & 0.120 & 0.325 & 0 & 0 & 1 \\
\hline Strong Madness Reputation, Leader B & 0.005 & 0.067 & 0 & 0 & 1 \\
\hline Slight Madness Reputation, Leader B & 0.120 & 0.325 & 0 & 0 & 1 \\
\hline Recent MID Initiations, Leader A & 0.507 & 0.787 & 0.200 & 0 & 4.800 \\
\hline Recent MID Initiations, Leader B & 0.507 & 0.787 & 0.200 & 0 & 4.800 \\
\hline Military Capabilities, State A & 0.031 & 0.049 & 0.007 & 0.000 & 0.208 \\
\hline Military Capabilities, State B & 0.031 & 0.049 & 0.007 & 0.000 & 0.208 \\
\hline \% Capabilities Held by State A & 0.500 & 0.425 & 0.500 & 0.00004 & 0.99996 \\
\hline Democracy, State A & 0.534 & 0.499 & 1 & 0 & 1 \\
\hline Democracy, State B & 0.534 & 0.499 & 1 & 0 & 1 \\
\hline Joint Democracy & 0.288 & 0.453 & 0 & 0 & 1 \\
\hline Land Contiguity & 0.151 & 0.358 & 0 & 0 & 1 \\
\hline Distance & 3.871 & 2.783 & 3.851 & 0.005 & 11.989 \\
\hline Dyad Length (Days) & 0.729 & 0.343 & 1 & 0.003 & 1.003 \\
\hline Peace Years & 39.614 & 36.886 & 33 & 0 & 194 \\
\hline
\end{tabular}

Table A3: Summary Statistics for Crisis Bargaining (MID Reciprocation) Dataset

\begin{tabular}{lllllc}
\hline Variable & Mean & SD & Median & Min & Max \\
\hline Reciprocation & 0.418 & 0.493 & 0 & 0 & 1 \\
Continuous Madness Rep., A & 0.065 & 0.352 & 0 & 0 & 3.457 \\
Continuous Madness Rep., B & 0.115 & 0.433 & 0 & 0 & 3.457 \\
Strong Madness Reputation, Leader A & 0.019 & 0.137 & 0 & 0 & 1 \\
Slight Madness Reputation, Leader A & 0.117 & 0.321 & 0 & 0 & 1 \\
Strong Madness Reputation, Leader B & 0.065 & 0.246 & 0 & 0 & 1 \\
Slight Madness Reputation, Leader B & 0.077 & 0.267 & 0 & 0 & 1 \\
Recent MID Initiations, Leader A & 0.803 & 0.898 & 0.500 & 0 & 4.400 \\
Recent MID Initiations, Leader B & 0.497 & 0.687 & 0.250 & 0 & 4.800 \\
Military Capabilities, State A & 0.026 & 0.045 & 0.007 & 0.000 & 0.208 \\
Military Capabilities, State B & 0.017 & 0.038 & 0.004 & 0.000 & 0.208 \\
\% Capabilities Held by State A & 0.584 & 0.305 & 0.635 & 0.002 & 0.9997 \\
Democracy, State A & 0.397 & 0.489 & 0 & 0 & 1 \\
Democracy, State B & 0.336 & 0.473 & 0 & 0 & 1 \\
Joint Democracy & 0.132 & 0.339 & 0 & 0 & 1 \\
Land Contiguity & 0.553 & 0.497 & 1 & 0 & 1 \\
Distance & 1.521 & 1.883 & 0.784 & 0.005 & 11.718 \\
First Act Hostility Level & 3.260 & 0.662 & 3 & 2 & 4 \\
\hline
\end{tabular}


Table A5: Observations with the Highest Values of Cook's D in the Initiation Regression

\begin{tabular}{llll}
\hline Leader A & \multicolumn{1}{c}{ Leader B } & Year & Cook's D \\
\hline Kim Il-Sung & Hosokawa & 1994 & 0.024 \\
Saddam Hussein & Kaifu & 1991 & 0.023 \\
Saddam Hussein & Khalifah Ath-Thani & 1991 & 0.023 \\
Saddam Hussein & Isa Ibn Al-Khalifah & 1991 & 0.022 \\
Saddam Hussein & Kohl & 1991 & 0.022 \\
Saddam Hussein & Jabir As-Sabah & 1999 & 0.021 \\
Saddam Hussein & Jabir As-Sabah & 1991 & 0.021 \\
Saddam Hussein & Jabir As-Sabah & 1992 & 0.021 \\
Bush & Milosevic & 1992 & 0.020 \\
Blair & Mugabe & 2002 & 0.020 \\
Major & Milosevic & 1992 & 0.020 \\
Bush & Saddam Hussein & 1991 & 0.020 \\
Mitterrand & Milosevic & 1992 & 0.020 \\
Bush & Saddam Hussein & 1992 & 0.020 \\
Mitsotakis & Milosevic & 1992 & 0.019 \\
Ibn Al-Khalifah & Saddam Hussein & 1994 & 0.019 \\
Clinton & Saddam Hussein & 1994 & 0.019 \\
Bush & Kim Jong-Il & 2004 & 0.019 \\
Bush & Ahmadinejad & 2007 & 0.019 \\
Schroder & Saddam Hussein & 1999 & 0.019 \\
\hline
\end{tabular}

Table A6: Observations with the Highest Values of Cook's D in the Reciprocation Regression

\begin{tabular}{|c|c|c|c|c|}
\hline Leader A & Leader B & Year & MID No. & Cook's D \\
\hline Kim Jong-II & Roh Moo Hyun & 2007 & 4479 & 0.014 \\
\hline Saddam Hussein & Jabir As-Sabah & 1999 & 4274 & 0.013 \\
\hline Saddam Hussein & Kaifu & 1991 & 3971 & 0.012 \\
\hline Ahmadinejad & al-Maliki & 2007 & 4536 & 0.011 \\
\hline Noriega & Reagan & 1989 & 3901 & 0.010 \\
\hline Ahmadinejad & al-Maliki & 2010 & 4547 & 0.010 \\
\hline Netanyahu & Saddam Hussein & 1998 & 4273 & 0.009 \\
\hline Ayatollah Khomeini & Reagan & 1988 & 2834 & 0.009 \\
\hline Obama & Hugo Chavez & 2010 & 4506 & 0.008 \\
\hline Bush & Saddam Hussein & 1991 & 3974 & 0.008 \\
\hline Bush & Saddam Hussein & 1992 & 3552 & 0.008 \\
\hline Howard & Saddam Hussein & 2003 & 4273 & 0.007 \\
\hline Deng Xiaoping & Gorbachev & 1986 & 2718 & 0.006 \\
\hline Alfonsin & Chiang Ching-Kuo & 1986 & 2579 & 0.006 \\
\hline Rafsanjani & Bush & 1991 & 3973 & 0.006 \\
\hline Deng Xiaoping & Yeltsin & 1994 & 4104 & 0.006 \\
\hline Ahmadinejad & Hamid Karzai & 2010 & 4599 & 0.005 \\
\hline Bush & Kim Jong-II & 2003 & 4455 & 0.005 \\
\hline Khatami & Bush & 2004 & 4519 & 0.005 \\
\hline Kim Il-Sung & Hosokawa & 1994 & 4022 & 0.005 \\
\hline
\end{tabular}


Table A7: Alternate Indicator Cutoffs and Dropping Outliers (Initiation Model)

\begin{tabular}{|c|c|c|c|c|}
\hline & $\begin{array}{c}(1) \\
\text { Top } 5 \% \text { Indicator } \\
\text { Cutoff }\end{array}$ & $\begin{array}{c}(2) \\
\text { Top } 20 \% \text { Indicator } \\
\text { Cutoff }\end{array}$ & $\begin{array}{c}\text { (3) } \\
\text { Top } 40 \% \text { Indicator } \\
\text { Cutoff }\end{array}$ & $\begin{array}{l}\quad(4) \\
\text { Cont. Measure, } \\
\text { Drop Top 16\% }\end{array}$ \\
\hline Strong Madness Rep, & $0.861^{\star \star \star}$ & 0.250 & 0.146 & \\
\hline Leader A & $(0.269)$ & $(0.181)$ & $(0.133)$ & \\
\hline Slight Madness Rep, & 0.066 & 0.086 & 0.099 & \\
\hline Leader A & $(0.067)$ & $(0.070)$ & $(0.072)$ & \\
\hline Strong Madness Rep, & $1.067^{\star \star \star}$ & $0.912^{\star \star \star}$ & $0.610^{\star \star \star}$ & \\
\hline Leader B & $(0.248)$ & $(0.124)$ & $(0.104)$ & \\
\hline Slight Madness Rep, & $0.297^{\star \star \star}$ & 0.128 & 0.144 & \\
\hline Leader B & $(0.070)$ & $(0.079)$ & $(0.089)$ & \\
\hline $\begin{array}{l}\text { Cont Madness Rep, } \\
\text { Leader A }\end{array}$ & & & & $\begin{array}{l}0.209^{\star \star} \\
(0.084)\end{array}$ \\
\hline Cont Madness Rep, & & & & $0.939^{\star * \star}$ \\
\hline Leader B & & & & $(0.295)$ \\
\hline Observations & 62384 & 62384 & 62384 & 62077 \\
\hline
\end{tabular}

Table A8: Address Regional and Time Bias (Initiation Model)

(1)

Region Fixed Effects

Strong Madness Rep,

Leader A

Slight Madness Rep,

Leader A

Strong Madness Rep,

Leader B

Slight Madness Rep,

Leader B

Observations

0.120
$(0.202)$
0.085
$(0.069)$
$0.919^{\star \star \star}$
$(0.144)$
$0.171^{\star \star}$
$(0.076)$
62384

0.120

0.085

$(0.069)$

$0.919^{\star * \star}$

$0.076)$

62384
(2) Time Fixed Effects

0.172

(0.210)

$0.123^{*}$

(0.069)

$0.904^{\star \star \star}$

$(0.138)$

$0.200^{\star \star *}$

(0.077)

Table A9: Address Pro-Western Bias and Strategic Use of Madness Adjectives (Initiation Model)

\begin{tabular}{|c|c|c|c|c|}
\hline & $\begin{array}{c}\text { (1) } \\
\text { Drop } \\
\text { Quotations }\end{array}$ & $\begin{array}{l}\text { (2) } \\
\text { Control for } \\
\text { US Affinity }\end{array}$ & $\begin{array}{l}\text { (3) } \\
\text { Only Non-US } \\
\text { Sources }\end{array}$ & $\begin{array}{c}(4) \\
\text { Drop English-Speaking } \\
\text { Western Countries }\end{array}$ \\
\hline Strong Madness Rep, & 0.327 & 0.190 & $0.364^{*}$ & 0.229 \\
\hline Leader A & $(0.220)$ & $(0.210)$ & $(0.200)$ & $(0.220)$ \\
\hline Slight Madness Rep, & $0.173^{\star \star}$ & 0.067 & $0.142^{\star \star}$ & -0.071 \\
\hline Leader A & $(0.073)$ & $(0.071)$ & $(0.072)$ & $(0.099)$ \\
\hline Strong Madness Rep, & $0.997^{\star \star \star}$ & $0.916^{\star \star \star}$ & $0.920^{\star \star \star}$ & $0.740^{\star \star \star}$ \\
\hline Leader B & $(0.170)$ & $(0.142)$ & $(0.150)$ & $(0.192)$ \\
\hline Slight Madness Rep, & $0.285^{\star \star \star}$ & $0.158^{\star \star}$ & $0.228^{\star \star \star}$ & 0.103 \\
\hline Leader B & $(0.079)$ & $(0.079)$ & $(0.077)$ & $(0.113)$ \\
\hline Affinity with US, & & 0.067 & & \\
\hline State A & & $(0.084)$ & & \\
\hline Affinity with US, & & -0.091 & & \\
\hline State B & & $(0.073)$ & & \\
\hline Observations & 62384 & 57730 & 62384 & 45980 \\
\hline
\end{tabular}


Table A10: Address Potentially Confounding Leader and Country Characteristics (Initiation Model)

\begin{tabular}{lcccc}
\hline & $\begin{array}{c}(1) \\
\text { Control for Time } \\
\text { in Office }\end{array}$ & $\begin{array}{c}(2) \\
\text { Drop Leaders in } \\
\text { Office <5 Years }\end{array}$ & $\begin{array}{c}(3) \\
\text { Matched Sample }\end{array}$ & $\begin{array}{c}\text { Control for Bluffing } \\
\text { Reputation }\end{array}$ \\
\hline Strong Madness Rep, & 0.181 & 0.282 & $-0.759^{\star \star}$ & 0.101 \\
Leader A & $(0.210)$ & $(0.248)$ & $(0.307)$ & $(0.207)$ \\
Slight Madness Rep, & 0.102 & 0.084 & 0.167 & 0.081 \\
Leader A & $(0.069)$ & $(0.090)$ & $(0.149)$ & $(0.069)$ \\
Strong Madness Rep, & $0.948^{\star \star \star}$ & $0.719^{\star \star \star}$ & $0.689^{\star \star \star}$ & $0.898^{\star \star \star}$ \\
Leader B & $(0.136)$ & $(0.169)$ & $(0.145)$ & $(0.137)$ \\
Slight Madness Rep, & $0.185^{\star \star}$ & 0.160 & 0.094 & $0.168^{\star \star}$ \\
Leader B & $(0.075)$ & $(0.098)$ & $(0.085)$ & $(0.076)$ \\
Years in Office, & $-0.011^{\star \star}$ & & & $-0.409^{\star \star \star}$ \\
Leader B & $(0.005)$ & & & $(0.095)$ \\
Recent Bluffs, & & & & -0.058 \\
Leader A & & & & $(0.093)$ \\
Recent Bluffs, & & & 35396 & 62384 \\
Leader B & & & & \\
Observations & 62384 & & & \\
\hline
\end{tabular}

Table A11: Adjustments to the Madness Measure (Initiation Model)

\begin{tabular}{|c|c|c|c|c|}
\hline & $\begin{array}{c}(1) \\
\text { Compare to Resolve } \\
\text { Reputation }\end{array}$ & $\begin{array}{c}\text { (2) } \\
\text { Drop Words Used } \\
\text { outside FP Context }\end{array}$ & $\begin{array}{c}(3) \\
\text { 5-Year } \\
\text { Average }\end{array}$ & $\begin{array}{c}(4) \\
\text { 10-Year } \\
\text { Average }\end{array}$ \\
\hline Strong Madness Rep, & 0.177 & 0.182 & 0.148 & 0.080 \\
\hline Leader A & $(0.208)$ & $(0.208)$ & $(0.142)$ & $(0.137)$ \\
\hline Slight Madness Rep, & 0.101 & $0.141^{\star \star}$ & -0.011 & -0.042 \\
\hline Leader A & $(0.069)$ & $(0.069)$ & $(0.064)$ & $(0.064)$ \\
\hline Strong Madness Rep, & $0.890^{\star \star \star}$ & $0.897^{\star \star \star}$ & $0.691^{\star \star \star}$ & $0.736^{\star \star \star}$ \\
\hline Leader B & $(0.136)$ & $(0.144)$ & $(0.152)$ & $(0.141)$ \\
\hline Slight Madness Rep, & $0.144^{\star}$ & $0.202^{\star \star \star}$ & $0.176^{\star \star}$ & $0.135^{\star}$ \\
\hline Leader B & $(0.076)$ & $(0.077)$ & $(0.069)$ & $(0.071)$ \\
\hline Strong Resolve Rep, & 0.051 & & & \\
\hline Leader B & $(0.137)$ & & & \\
\hline Slight Resolve Rep, & 0.073 & & & \\
\hline Leader B & $(0.076)$ & & & \\
\hline Observations & 62384 & 62384 & 62384 & 62384 \\
\hline
\end{tabular}

Table A12: Different Sample and Dependent Variables (Initiation Model)

\begin{tabular}{lcccc}
\hline & $\begin{array}{c}(1) \\
\text { No Politically } \\
\text { Relevant Restriction }\end{array}$ & $\begin{array}{c}(2) \\
\text { Only Dyads with a } \\
\text { MID in Last 15 Years }\end{array}$ & $\begin{array}{c}\text { (3) } \\
\text { Forceful } \\
\text { MID DV }\end{array}$ & $\begin{array}{c}(4) \\
\text { Fatal } \\
\text { MID DV }\end{array}$ \\
\hline Strong Madness Rep, & 0.160 & -0.264 & -0.008 & 0.308 \\
Leader A & $(0.130)$ & $(0.199)$ & $(0.249)$ & $(0.210)$ \\
Slight Madness Rep, & $0.217^{\star \star \star}$ & 0.013 & 0.023 & -0.166 \\
Leader A & $(0.061)$ & $(0.074)$ & $(0.094)$ & $(0.107)$ \\
Strong Madness Rep, & $0.899^{\star \star \star}$ & $0.524^{\star \star \star}$ & $0.784^{\star \star \star}$ & $0.810^{\star \star \star}$ \\
Leader B & $(0.079)$ & $(0.128)$ & $(0.149)$ & $(0.156)$ \\
Slight Madness Rep, & $0.294^{\star \star \star}$ & $0.133^{\star}$ & 0.096 & 0.032 \\
Leader B & $(0.067)$ & $(0.079)$ & $(0.098)$ & $(0.120)$ \\
Observations & 605264 & 11092 & 62384 & 62384 \\
\hline
\end{tabular}


Table A13: Interaction (Initiation Model)

\begin{tabular}{lc}
\hline Strong Madness Rep, & 0.177 \\
Leader A & $(0.208)$ \\
Strong Madness Rep, & $0.904^{\star \star \star}$ \\
Leader B & $(0.140)$ \\
Slight Madness Rep, & 0.052 \\
Leader A & $(0.074)$ \\
Slight Madness Rep, & 0.103 \\
Leader B & $(0.084)$ \\
Slight Madness Rep A & $0.438^{\star}$ \\
X Slight Madness Rep B & $(0.227)$ \\
Observations & 62384 \\
\hline
\end{tabular}

Table A14: Alternate Indicator Cutoffs and Dropping Outliers (Recip Model)

\begin{tabular}{|c|c|c|c|c|}
\hline & $\begin{array}{c}(1) \\
\text { Top 5\% } \\
\text { Indicator Cutoff }\end{array}$ & $\begin{array}{c}\text { (2) } \\
\text { Top } 10 \% \\
\text { Indicator Cutoff }\end{array}$ & $\begin{array}{c}\text { (3) } \\
\text { Top 20\% } \\
\text { Indicator Cutoff }\end{array}$ & $\begin{array}{c}(4) \\
\text { Cont. Measure, } \\
\text { Dropping Top 1\% }\end{array}$ \\
\hline Strong Madness Rep, & $0.665^{\star \star \star}$ & $0.686^{\star \star \star}$ & 0.249 & \\
\hline Leader A & $(0.215)$ & $(0.218)$ & $(0.177)$ & \\
\hline Slight Madness Rep, & -0.194 & -0.189 & -0.159 & \\
\hline Leader A & $(0.208)$ & $(0.218)$ & $(0.226)$ & \\
\hline Strong Madness Rep, & $0.647^{\star}$ & -0.288 & $-0.616^{\star \star \star}$ & \\
\hline Leader B & $(0.389)$ & $(0.220)$ & $(0.198)$ & \\
\hline Slight Madness Rep, & $-0.495^{\star \star \star}$ & $-0.372^{\star \star}$ & -0.072 & \\
\hline Leader B & $(0.154)$ & $(0.171)$ & $(0.206)$ & \\
\hline Continuous Madness & & & & 0.099 \\
\hline Rep, A & & & & $(0.116)$ \\
\hline Continuous Madness & & & & 0.034 \\
\hline Rep, B & & & & $(0.123)$ \\
\hline Observations & 759 & 759 & 759 & 753 \\
\hline
\end{tabular}

Table A15: Address Regional and Time Bias (Recip Model)

(1)

Region Fixed Effects

Strong Madness Rep,

Leader A

Slight Madness Rep,

Leader A

Strong Madness Rep,

Leader B

Slight Madness Rep,

Leader B

Observations

$0.278^{\star \star}$
$(0.133)$
-0.141
$(0.198)$
$-0.542^{\star \star}$
$(0.225)$
-0.251
$(0.176)$
759

(2) Time Fixed Effects 
Table A16: Address Pro-Western Bias and Strategic Use of Madness Adjectives (Recip Model)

\begin{tabular}{lcccc}
\hline & $\begin{array}{c}(1) \\
\text { Drop } \\
\text { Quotations }\end{array}$ & $\begin{array}{c}(2) \\
\text { Control for } \\
\text { US Affinity }\end{array}$ & $\begin{array}{c}(3) \\
\text { Only Non-US } \\
\text { Sources }\end{array}$ & $\begin{array}{c}(4) \\
\text { Drop English-Speaking } \\
\text { Western Countries }\end{array}$ \\
\hline Strong Madness Rep, & 0.219 & $0.408^{\star \star \star}$ & $0.430^{\star}$ & $0.492^{\star \star \star}$ \\
Leader A & $(0.250)$ & $(0.143)$ & $(0.247)$ & $(0.148)$ \\
Slight Madness Rep, & -0.097 & -0.023 & -0.157 & -0.233 \\
Leader A & $(0.192)$ & $(0.195)$ & $(0.230)$ & $(0.222)$ \\
Strong Madness Rep, & $-0.482^{\star \star}$ & $-0.447^{\star \star}$ & -0.252 & $-0.653^{\star \star}$ \\
Leader B & $(0.231)$ & $(0.202)$ & $(0.230)$ & $-0.272)$ \\
Slight Madness Rep, & $-0.339^{\star \star}$ & -0.283 & $-0.371^{\star \star}$ & $\left(0.296^{\star \star}\right.$ \\
Leader B & $(0.164)$ & $(0.177)$ & $(0.175)$ & \\
Affinity with US, & & $-0.403^{\star}$ & & \\
State A & & $(0.237)$ & & \\
Affinity with US, & & 0.008 & 759 & 651 \\
State B & & $(0.202)$ & & \\
Observations & 759 & 723 & & \\
\hline
\end{tabular}

Table A17: Address Potentially Confounding Leader and Country Characteristics (Recip Model)

\begin{tabular}{|c|c|c|c|c|c|}
\hline & $\begin{array}{c}(1) \\
\text { Control for } \\
\text { Years in Office }\end{array}$ & $\begin{array}{c}(2) \\
\text { Drop Leaders } \\
\text { in Office }<5 \text { Years }\end{array}$ & $\begin{array}{c}\text { (3) } \\
\text { Matched } \\
\text { Sample }\end{array}$ & $\begin{array}{l}(4) \\
\text { Drop Strategic } \\
\text { Blunders }\end{array}$ & $\begin{array}{c}\text { (5) } \\
\text { Control for } \\
\text { Bluffing Reputation }\end{array}$ \\
\hline Strong Madness Rep, & $0.435^{\star \star \star}$ & $0.439^{\star \star \star}$ & $0.749^{\star}$ & $0.450^{\star \star \star}$ & $0.289^{*}$ \\
\hline Leader A & $(0.160)$ & $(0.165)$ & $(0.447)$ & $(0.162)$ & $(0.156)$ \\
\hline Slight Madness Rep, & -0.178 & $-0.527^{\star}$ & 0.334 & -0.073 & -0.160 \\
\hline Leader A & $(0.223)$ & $(0.274)$ & $(0.336)$ & $(0.226)$ & $(0.194)$ \\
\hline Strong Madness Rep, & $-0.563^{\star \star \star}$ & $-0.900^{* *}$ & -0.476 & $-0.624^{\star \star \star}$ & $-0.619^{\star \star \star}$ \\
\hline Leader B & $(0.201)$ & $(0.442)$ & $(0.524)$ & $(0.203)$ & $(0.201)$ \\
\hline Slight Madness Rep, & -0.210 & 0.027 & -0.005 & -0.203 & -0.192 \\
\hline Leader B & $(0.183)$ & $(0.221)$ & $(0.284)$ & $(0.191)$ & $(0.182)$ \\
\hline Years in Office, & 0.000 & & & & \\
\hline Leader A & $(0.011)$ & & & & \\
\hline Recent Bluffs, & & & & & $-0.515^{\star}$ \\
\hline Leader $A$ & & & & & $(0.299)$ \\
\hline Recent Bluffs, & & & & & $-0.308^{\star}$ \\
\hline Leader B & & & & & $(0.184)$ \\
\hline Observations & 759 & 449 & 347 & 707 & 759 \\
\hline
\end{tabular}

Table A18: Adjustments to the Madness Measure (Recip Model)

\begin{tabular}{|c|c|c|c|c|}
\hline & $\begin{array}{c}(1) \\
\text { Compare to Resolve } \\
\text { Reputation }\end{array}$ & $\begin{array}{c}\text { (2) } \\
\text { Drop Words Used } \\
\text { outside FP Context }\end{array}$ & $\begin{array}{c}\text { (3) } \\
\text { 5-Year Average }\end{array}$ & $\begin{array}{c}\text { (4) } \\
\text { 10-Year Average }\end{array}$ \\
\hline Strong Madness Rep, & $0.400^{\star \star}$ & $0.703^{\star \star \star}$ & 0.072 & -0.060 \\
\hline Leader A & $(0.160)$ & $(0.222)$ & $(0.167)$ & $(0.165)$ \\
\hline Slight Madness Rep, & -0.256 & -0.180 & -0.245 & -0.254 \\
\hline Leader A & $(0.223)$ & $(0.247)$ & $(0.188)$ & $(0.183)$ \\
\hline Strong Resolve Rep, & $0.551^{*}$ & & & \\
\hline Leader A & $(0.325)$ & & & \\
\hline Slight Resolve Rep, & 0.026 & & & \\
\hline Leader A & $(0.245)$ & & & \\
\hline Strong Madness Rep, & $-0.584^{\star \star \star}$ & -0.343 & $-0.328^{*}$ & $-0.343^{*}$ \\
\hline Leader B & $(0.202)$ & $(0.219)$ & $(0.191)$ & $(0.190)$ \\
\hline Slight Madness Rep, & -0.208 & -0.243 & $-0.559^{\star * \star}$ & -0.113 \\
\hline Leader B & $(0.191)$ & $(0.179)$ & $(0.146)$ & $(0.139)$ \\
\hline Observations & 759 & 759 & 759 & 759 \\
\hline
\end{tabular}


Table A19: Dropping Some MIDs (Recip Model)

\begin{tabular}{lccc}
\hline & $\begin{array}{c}(1) \\
\text { Drop MIDs Beginning } \\
\text { with Force }\end{array}$ & $\begin{array}{c}(2) \\
\text { Drop Non-Revisionist } \\
\text { MIDs }\end{array}$ & $\begin{array}{c}(3) \\
\text { Retain Only One Observation } \\
\text { per MID Target }\end{array}$ \\
\hline Strong Madness Rep, & $0.386^{\star \star}$ & -0.313 & $0.425^{\star \star \star}$ \\
Leader A & $(0.181)$ & $(0.445)$ & $(0.162)$ \\
Slight Madness Rep, & -0.317 & 0.046 & -0.308 \\
Leader A & $(0.254)$ & $(0.192)$ & $(0.272)$ \\
Strong Madness Rep, & $-0.510^{\star \star}$ & $-0.726^{\star \star \star}$ & -0.240 \\
Leader B & $(0.208)$ & $(0.249)$ & $(0.196)$ \\
Slight Madness Rep, & -0.211 & -0.434 & -0.145 \\
Leader B & $(0.228)$ & $(0.265)$ & $(0.231)$ \\
Observations & 468 & 491 & 644 \\
\hline
\end{tabular}

Table A20: Interaction (Recip Model)

\begin{tabular}{lc}
\hline Strong Madness Rep, & $0.436^{\star \star *}$ \\
Leader A & $(0.156)$ \\
Strong Madness Rep, & $-0.563^{\star \star \star}$ \\
Leader B & $(0.201)$ \\
Slight Madness Rep, & -0.174 \\
Leader A & $(0.218)$ \\
Slight Madness Rep, & -0.204 \\
Leader B & $(0.232)$ \\
Slight Madness Rep A & -0.031 \\
X Slight Madness Rep B & $(0.434)$ \\
Observations & 759 \\
\hline
\end{tabular}

Table A21: Conflict Selection Model (Recip Model)

\begin{tabular}{lc}
\hline Strong Madness Rep, & $0.850^{\star \star \star}$ \\
Leader A & $(0.249)$ \\
Slight Madness Rep, & -0.226 \\
Leader A & $(0.290)$ \\
Strong Madness Rep, & -0.211 \\
Leader B & $(0.276)$ \\
Slight Madness Rep, & -0.302 \\
Leader B & $(0.214)$ \\
Rho & -0.078 \\
Observations & $(0.227)$ \\
\hline
\end{tabular}


Table A22: Interactions with Relative Capabilities

\begin{tabular}{|c|c|c|}
\hline & $\begin{array}{c}(1) \\
\text { Deterrence }\end{array}$ & $\begin{array}{c}(2) \\
\text { Crisis Bargaining }\end{array}$ \\
\hline Strong Madness Rep, & 0.176 & -0.363 \\
\hline Leader A & $(0.210)$ & $(0.417)$ \\
\hline Slight Madness Rep, & 0.096 & $-1.063^{\star \star}$ \\
\hline Leader A & $(0.069)$ & $(0.534)$ \\
\hline Strong Madness Rep, & 0.437 & $-0.565^{\star \star \star}$ \\
\hline Leader B & $(0.365)$ & $(0.203)$ \\
\hline Slight Madness Rep, & -0.030 & -0.196 \\
\hline Leader B & $(0.117)$ & $(0.188)$ \\
\hline$\%$ of Military Capabilities & $0.333^{\star \star \star}$ & -0.234 \\
\hline Held by $A$ & $(0.099)$ & $(0.247)$ \\
\hline Strong Madness Rep A & & $1.302^{\star \star}$ \\
\hline X \% Capabilities & & $(0.551)$ \\
\hline Slight Madness Rep A & & $1.244^{\star}$ \\
\hline X \% Capabilities & & $(0.703)$ \\
\hline Strong Madness Rep B & 0.662 & \\
\hline X \% Capabilities & $(0.459)$ & \\
\hline Slight Madness Rep B & $0.506^{\star \star}$ & \\
\hline X \% Capabilities & $(0.209)$ & \\
\hline Observations & 62384 & 759 \\
\hline
\end{tabular}

Table A23: Tests Mentioned in Footnotes (Initiation Model)

\begin{tabular}{lcccc}
\hline & $\begin{array}{c}(1) \\
\text { Retain Tiny } \\
\text { Countries }\end{array}$ & $\begin{array}{c}(2) \\
\text { Drop Extreme } \\
\text { Outlier }\end{array}$ & $\begin{array}{c}(3) \\
\text { Count Recent } \\
\text { MIDs by Country }\end{array}$ & $\begin{array}{c}(4) \\
\text { Count Only Losing } \\
\text { Recent MIDs by Leader }\end{array}$ \\
\hline Strong Madness Rep, & 0.170 & 0.179 & 0.139 & 0.231 \\
Leader A & $(0.208)$ & $(0.209)$ & $(0.211)$ & $(0.207)$ \\
Slight Madness Rep, & 0.094 & 0.099 & $0.194^{\star \star \star}$ & $0.238^{\star \star \star}$ \\
Leader A & $(0.069)$ & $(0.069)$ & $(0.067)$ & $(0.069)$ \\
Strong Madness Rep, & $0.912^{\star \star \star}$ & $0.909^{\star \star \star}$ & $0.857^{\star \star \star}$ & $0.902^{\star \star \star}$ \\
Leader B & $(0.140)$ & $(0.140)$ & $(0.137)$ & $(0.139)$ \\
Slight Madness Rep, & $0.172^{\star \star}$ & $0.170^{\star \star}$ & $0.180^{\star \star}$ & $0.207^{\star \star \star}$ \\
Leader B & $(0.075)$ & $(0.075)$ & $(0.075)$ & $(0.076)$ \\
Observations & 67522 & 62366 & 62384 & 62384 \\
\hline
\end{tabular}

Table A24: More Tests Mentioned in Footnotes (Initiation Model)

\begin{tabular}{lcc}
\hline & $(1)$ & $(2)$ \\
& Minimalist Model & Logged Madness Measure \\
\hline Strong Madness Rep, & 0.191 & \\
Leader A & $(0.209)$ & 0.057 \\
Slight Madness Rep, & $(0.070)$ & $0.855^{\star \star \star}$ \\
Leader A & $(0.139)$ & $0.362^{\star \star}$ \\
Strong Madness Rep, & $0.163^{\star \star}$ & $(0.184)$ \\
Leader B & $(0.073)$ & $0.917^{\star \star \star}$ \\
Slight Madness Rep, & & $(0.138)$ \\
Leader B & & 62384 \\
Logged Continuous & & 62384 \\
Madness Rep, A & & \\
Logged Continuous & & \\
Madness Rep, B & & \\
Observations & & \\
\hline
\end{tabular}


Table A25: Tests Mentioned in Footnotes (Recip Model)

\begin{tabular}{lccc}
\hline & $(1)$ & $(2)$ & $(3)$ \\
& $\begin{array}{c}\text { Retain Tiny } \\
\text { Countries }\end{array}$ & $\begin{array}{c}\text { Count All } \\
\text { Recent MIDs }\end{array}$ & $\begin{array}{c}\text { Count Only Recent Losing MIDs } \\
\text { Initiated by Leader }\end{array}$ \\
\hline Strong Madness Rep, & $0.457^{\star \star \star}$ & $0.242^{\star \star}$ & $0.424^{\star \star \star}$ \\
Leader A & $(0.145)$ & $(0.122)$ & $(0.164)$ \\
Slight Madness Rep, & -0.158 & -0.236 & -0.210 \\
Leader A & $(0.217)$ & $(0.202)$ & $(0.202)$ \\
Strong Madness Rep, & $-0.536^{\star \star \star}$ & $-0.709^{\star \star \star}$ & $-0.557^{\star \star \star}$ \\
Leader B & $(0.201)$ & $(0.198)$ & $(0.198)$ \\
Slight Madness Rep, & -0.198 & -0.270 & -0.196 \\
Leader B & $(0.182)$ & $(0.184)$ & $(0.190)$ \\
Observations & 773 & 759 & 759 \\
\hline
\end{tabular}

Table A26: More Tests Mentioned in Footnotes (Recip Model)

\begin{tabular}{|c|c|c|c|c|}
\hline & $\begin{array}{c}(1) \\
\text { Minimalist Model }\end{array}$ & $\begin{array}{c}(2) \\
\text { Logged Madness Measure }\end{array}$ & $\begin{array}{c}\text { (3) } \\
\text { Cluster by Leader A }\end{array}$ & Cluster by Country B \\
\hline $\begin{array}{l}\text { Strong Madness Rep, } \\
\text { Leader A }\end{array}$ & $\begin{array}{l}0.418^{\star \star \star} \\
(0.153)\end{array}$ & & $\begin{array}{l}0.436^{\star \star} \\
(0.209)\end{array}$ & $\begin{array}{c}0.436 \\
(0.358)\end{array}$ \\
\hline Slight Madness Rep, & -0.128 & & -0.178 & $\begin{array}{l}(0.050) \\
-0.178\end{array}$ \\
\hline Leader A & $(0.234)$ & & $(0.209)$ & $(0.190)$ \\
\hline Strong Madness Rep, & $-0.516^{\star \star \star}$ & & $-0.563^{* *}$ & -0.563 \\
\hline Leader B & $(0.198)$ & & $(0.246)$ & $(0.381)$ \\
\hline Slight Madness Rep, & -0.190 & & -0.210 & -0.210 \\
\hline Leader B & $(0.190)$ & & $(0.172)$ & $(0.214)$ \\
\hline Logged Continuous & & $0.416^{\star \star}$ & & \\
\hline Madness Rep, A & & $(0.162)$ & & \\
\hline Logged Continuous & & -0.184 & & \\
\hline Madness Rep, B & & $(0.242)$ & & \\
\hline Observations & 759 & 759 & 759 & 759 \\
\hline
\end{tabular}

\section{Reference}

McManus, R.W. (2019) Crazy Like a Fox? Are Leaders with Reputations for Madness More Successful at International Coercion? British Journal of Political Science. Published by Cambridge University Press, 9 December 2019. doi: $10.1017 /$ S0007123419000401.

Cite this article: McManus RW (2021). Crazy Like a Fox? Are Leaders with Reputations for Madness More Successful at International Coercion? - CORRIGENDUM. British Journal of Political Science 51, 294-307. https://doi.org/10.1017/ S000712342000040X 